\begin{tabular}{|c|c|}
\hline \multicolumn{2}{|c|}{ Statistica Sinica Preprint No: SS-2020-0226 } \\
\hline Title & $\begin{array}{l}\text { Feature-Weighted Elastic Net: Using "Features of } \\
\text { Features" for Better Prediction }\end{array}$ \\
\hline Manuscript ID & SS-2020-0226 \\
\hline URL & http://www.stat.sinica.edu.tw/statistica/ \\
\hline DOI & $10.5705 /$ ss. 202020.0226 \\
\hline Complete List of Authors & $\begin{array}{l}\text { Jingyi Kenneth Tay, } \\
\text { Nima Aghaeepour, } \\
\text { Trevor Hastie and } \\
\text { Robert Tibshirani }\end{array}$ \\
\hline Corresponding Author & Jingyi Kenneth Tay \\
\hline E-mail & kjytay@stanford.edu \\
\hline tice: Accepted version subj & ct to English editing. \\
\hline
\end{tabular}


Statistica Sinica

\title{
FEATURE-WEIGHTED ELASTIC NET: USING "FEATURES OF FEATURES" FOR "BETTER PREDICTION
}

\author{
J. Kenneth Tay ${ }^{1}$, Nima Aghaeepour ${ }^{2,3,4}$, Trevor Hastie ${ }^{1,4}$
}

and Robert Tibshirani ${ }^{1,4}$

${ }^{1}$ Department of Statistics, Stanford University

${ }^{2}$ Department of Anesthesiology, Pain, and Perioperative Medicine, Stanford University

${ }^{3}$ Department of Pediatrics, Stanford University

${ }^{4}$ Department of Biomedical Data Sciences, Stanford University

Abstract: In some supervised learning settings, the practitioner might have additional information on the features used for prediction. We propose a new method which leverages this additional information for better prediction. The method, which we call the feature-weighted elastic net ( "fwelnet"), uses these "features of features" to adapt the relative penalties on the feature coefficients in the elastic net penalty. In our simulations, fwelnet outperforms the lasso in terms of test mean squared error and usually gives an improvement in true positive rate or false positive rate for feature selection. We present connections between this method and the group lasso, and also to Bayesian estimation. We also apply this method to early prediction of preeclampsia, where fwelnet outperforms the lasso in terms 
of 10 -fold cross-validated area under the curve (0.84 vs. 0.80$)$, and suggest how fwelnet might be used for multi-task learning.

Key words and phrases: Model selection/variable selection, feature information, prediction.

\section{Introduction}

Consider the usual linear regression model: given $n$ realizations of $p$ pre-

dictors $\mathbf{X}=\left\{x_{i j}\right\}$ for $i=1,2, \ldots, n$ and $j=1,2, \ldots, p$, the response $\mathbf{y}=\left(y_{1}, \ldots, y_{n}\right)$ is modeled as

$$
y_{i}=\beta_{0}+\sum_{j=1}^{p} x_{i j} \beta_{j}+\epsilon_{i}
$$

with $\epsilon$ having mean 0 and variance $\sigma^{2}$. The ordinary least squares (OLS) estimates of $\beta_{j}$ are obtained by minimizing the residual sum of squares (RSS). There has been much work on regularized estimators that offer an advantage over the OLS estimates, both in terms of prediction accuracy and interpretation of the fitted model. One popular regularized estimator is the elastic net (Zou and Hastie, 2005). Letting $\beta=\left(\beta_{1}, \ldots, \beta_{p}\right)^{T}$, the elastic net minimizes the objective function

$$
J\left(\beta_{0}, \beta\right)=\frac{1}{2}\left\|\mathbf{y}-\beta_{0} \mathbf{1}-\mathbf{X} \beta\right\|_{2}^{2}+\lambda\left[\alpha\|\beta\|_{1}+\frac{1-\alpha}{2}\|\beta\|_{2}^{2}\right] .
$$

The elastic net has two tuning parameters: $\lambda \geq 0$ which controls the 
overall sparsity of the solution, and $\alpha \in[0,1]$ which determines the relative weight of the $\ell_{1}$ and $\ell_{2}$-squared penalties. Setting $\alpha=0$ corresponds to ridge regression (Hoerl and Kennard, 1970), while $\alpha=1$ corresponds to the lasso (Tibshirani, 1996). One reason for the elastic net's popularity is its computational efficiency: $J$ is convex in its parameters, so solutions can be found efficiently even for very large $n$ and $p$. In addition, the solution for an entire path of $\lambda$ values can be computed quickly using warm starts (Friedman et al., 2010).

In some settings, we have information about the features themselves. For example, in genomics, we know that each gene belongs to one or more genetic pathways, and we may expect genes in the same pathway to have correlated effects on the response. Methods which leverage such information are likely to perform better prediction and inference than methods which ignore it. However, many popular methods, including the elastic net, do not use such information in the model-fitting process.

In this paper, we develop a framework for organizing such feature information and propose a variant of the elastic net which uses this information in model-fitting. We assume that the feature information is quantitative, allowing us to think of each source as a "feature" of the features. For example, in the genomics setting, the $k$ th source of information could be the 
indicator variable for whether the $j$ th feature belongs to the $k$ th genetic pathway. We organize these "features of features" into an auxiliary matrix $\mathbf{Z} \in \mathbb{R}^{p \times K}$, where $p$ is the number of features and $K$ is the number of sources of feature information. Let $\mathbf{z}_{j} \in \mathbb{R}^{K}$ denote the $j$ th row of $\mathbf{Z}$ as a column vector. We propose assigning each feature a score $\mathbf{z}_{j}^{T} \theta$, i.e. a linear combination of its "features of features", and using these scores to influence the penalty weight in the elastic net penalty:

$$
J_{\lambda, \alpha, \theta}\left(\beta_{0}, \beta\right)=\frac{1}{2}\left\|\mathbf{y}-\beta_{0} \mathbf{1}-\mathbf{X} \beta\right\|_{2}^{2}+\lambda \sum_{j=1}^{p} w_{j}(\theta)\left[\alpha\left|\beta_{j}\right|+\frac{1-\alpha}{2} \beta_{j}^{2}\right],
$$

where $w_{j}(\theta)=f\left(\mathbf{z}_{j}^{T} \theta\right)$ for some function $f$. $\theta$ is a hyperparameter in $\mathbb{R}^{K}$ which the algorithm needs to select. In the final model, $\mathbf{z}_{j}^{T} \theta$ can be thought of as an indication of how influential feature $j$ is on the response.

The rest of this paper is organized as follows. In Section 2, we survey past work on incorporating "features of features" in supervised learning. In Section 3, we propose a method, the feature-weighted elastic net ("fwelnet"), which uses the scores in model-fitting. We present connections to the group lasso and Bayesian estimation in Section 4, and illustrate fwelnet's performance on simulated data in Section 5 and on a real data example in Section 6. In Section 7, we show how fwelnet can be used in multi-task learning. We end with a discussion and ideas for future work. The appendix contains further details and proofs. 


\section{Related work}

The idea of assigning different penalty weights for features in the lasso or elastic net objective is not new. The adaptive lasso (Zou, 2006) assigns feature $j$ a penalty weight $w_{j}=1 /\left|\hat{\beta}_{j}^{O L S}\right|^{\gamma}$, where $\hat{\beta}_{j}^{O L S}$ is the estimated OLS coefficent for feature $j$ and $\gamma>0$ is some hyperparameter. However, the OLS solution only depends on $\mathbf{X}$ and $\mathbf{y}$ and does not incorporate any external information. In the work closest to ours, Bergersen et al. (2011) propose using weights $w_{j}=\frac{1}{\left|\eta_{j}(\mathbf{y}, \mathbf{X}, \mathbf{Z})\right|^{q}}$, where $\eta_{j}$ is some function (possibly varying for $j$ ) and $q$ is a hyperparameter controlling the shape of the weight function. While the authors present two ideas for what the $\eta_{j}$ 's could be, they do not give general guidance on how to choose these functions which could drastically influence the model-fitting algorithm.

There is a correspondence between penalized regression estimates and Bayesian maximum a posteriori (MAP) estimates with a particular prior for the coefficients. Within this Bayesian framework, some methods propose using external feature information to guide the choice of prior. For example, van de Wiel et al. (2016) take an empirical Bayes approach to estimate the prior for ridge regression, while Velten and Huber (2018) use variational Bayes to do so for general convex penalties.

Most previous approaches for penalized regression with external infor- 
mation on the features only work with specific types of such information. Several methods have been developed to make use of feature grouping information. Popular methods include the group lasso (Yuan and Lin, 2006) and the overlap group lasso (Jacob et al., 2009). IPF-LASSO (integrative lasso with penalty factors) (Boulesteix et al. 2017) gives each group its own penalty parameter, to be chosen via cross-validation. Tai and Pan (2007) modify the penalized partial least squares (PLS) and nearest shrunken centroids methods to have group-specific penalties.

Other methods have been developed to incorporate "network-like" or feature similarity information. The fused lasso (Tibshirani et al., 2005) adds an $\ell_{1}$ penalty on the successive differences of the coefficients to impose smoothness on the coefficient profile. Structured elastic net Slawski et al. 2010) generalizes the fused lasso by replacing the $\ell_{2}$-squared penalty in elastic net with $\beta^{T} \Lambda \beta$, where $\Lambda$ is a symmetric, positive semi-definite matrix chosen to reflect some a priori known structure between the features. Li and $\mathrm{Li}(2008)$ is a special case of structured elastic net, where $\Lambda$ is equal to the normalized Laplacian matrix of the feature network graph. Mollaysa et al. (2017) use the feature information matrix $\mathbf{Z}$ to compute a feature similarity matrix, which in turn is used to construct a penalty term in the loss criterion. We note that their approach implicitly assumes that the 
sources of feature information are equally relevant, which may or may not be the case.

It is not clear how most of the prior work can be generalized to generic sources of feature information. Our method has the distinction of being able to work directly with real-valued feature information and to integrate multiple sources of feature information. While van de Wiel et al. (2016) claim to be able to handle binary, nominal, ordinal and continuous feature information, the method actually ranks and groups features based on such information and only uses this grouping information. Nevertheless, it is able to incorporate more than one source of feature information.

\section{Feature-weighted elastic net ("fwelnet")}

One way to utilize the scores $\mathbf{z}_{j}^{T} \theta$ in model-fitting is to give each feature a different penalty weight in the elastic net objective based on its score:

$$
J_{\lambda, \alpha, \theta}\left(\beta_{0}, \beta\right)=\frac{1}{2}\left\|\mathbf{y}-\beta_{0} \mathbf{1}-\mathbf{X} \beta\right\|_{2}^{2}+\lambda \sum_{j=1}^{p} w_{j}(\theta)\left[\alpha\left|\beta_{j}\right|+\frac{1-\alpha}{2} \beta_{j}^{2}\right],
$$

where $w_{j}(\theta)=f\left(\mathbf{z}_{j}^{T} \theta\right)$ for some function $f$. Our proposed method, which we call the feature-weighted elastic net ("fwelnet"), specifies $f$ :

$$
w_{j}(\theta)=\frac{\sum_{\ell=1}^{p} \exp \left(\mathbf{z}_{\ell}^{T} \theta\right)}{p \exp \left(\mathbf{z}_{j}^{T} \theta\right)}
$$

The fwelnet algorithm seeks the minimizer of this objective function 
over $\beta_{0}$ and $\beta$ :

$$
\left(\hat{\beta}_{0}, \hat{\beta}\right)=\underset{\beta_{0}, \beta}{\operatorname{argmin}} \frac{1}{2}\left\|\mathbf{y}-\beta_{0} \mathbf{1}-\mathbf{X} \beta\right\|_{2}^{2}+\lambda \sum_{j=1}^{p} w_{j}(\theta)\left[\alpha\left|\beta_{j}\right|+\frac{1-\alpha}{2} \beta_{j}^{2}\right] .
$$

There are a number of reasons for this choice of penalty factors. First, when $\theta=0$, we have $w_{j}(\theta)=1$ for all $j$, reducing fwelnet to the original elastic net. Second, $w_{j}(\theta) \geq 1 / p$ for all $j$ and $\theta$, ensuring that we do not end up with features having negligible penalty. This allows the fwelnet solution to have a wider range of sparsity across $\lambda$ hyperparameter values. Third, this formulation provides theoretical connections which we detail in Section 4. Finally, a feature's score has a natural interpretation: if $\mathbf{z}_{j}^{T} \theta$ is relatively large, then $w_{j}$ is relatively small, meaning that feature $j$ is more important for the response and hence should have smaller penalty.

We illustrate the last property via a simulated example. In this simulation, we have $n=200$ observations and $p=100$ features which come in groups of 10 . The response is a linear combination of the first two groups with additive Gaussian noise. The coefficient for the first group is 4 while the coefficient for the second group is -2 so that the first group exhibits stronger correlation to the response compared to the second group. The "features of features" matrix $\mathbf{Z} \in \mathbb{R}^{100 \times 10}$ is grouping information, i.e. $z_{j k}=1\{$ feature $j$ belongs to group $k\}$. Figure 1 shows the penalty factors 
3.1 Computing the fwelnet solution9

$w_{j}$ that fwelnet assigns the features. (The hyperparameter $\theta$ was determined using Algorithm 1 described in Section 3.1.) As one would expect, the features in the first group have the smallest penalty factor followed by features in the second group. In contrast, the original elastic net algorithm would assign penalty factors $w_{j}=1$ for all $j$.

\subsection{Computing the fwelnet solution}

It can be easily shown that $\hat{\beta}_{0}=\overline{\mathbf{y}}-\sum_{j=1}^{p} \hat{\beta}_{j} \overline{\mathbf{x}}_{. j}$. Henceforth, we assume that $\mathbf{y}$ and the columns of $\mathbf{X}$ are centered so that $\hat{\beta}_{0}=0$ and we can ignore the intercept term in the rest of the discussion.

For given values of $\lambda, \alpha$ and $\theta$, it is easy to solve $(3.2)$ : the objective function is convex in $\beta$ and $\hat{\beta}$ can be found efficiently using algorithms such as coordinate descent. However, to deploy fwelnet in practice we need to determine the hyperparameter values $\hat{\lambda} \in \mathbb{R}, \hat{\alpha} \in \mathbb{R}$ and $\hat{\theta} \in \mathbb{R}^{K}$ that give good performance. When $K$, the number of sources of feature information, is small, one could run the algorithm for a grid of $\theta$ values, then pick the value which gives the smallest cross-validated loss. Unfortunately, this approach is computationally infeasible for even moderate values of $K$.

To avoid this computational bottleneck, we propose solving the mini- 


\section{Penalty weight for each feature}

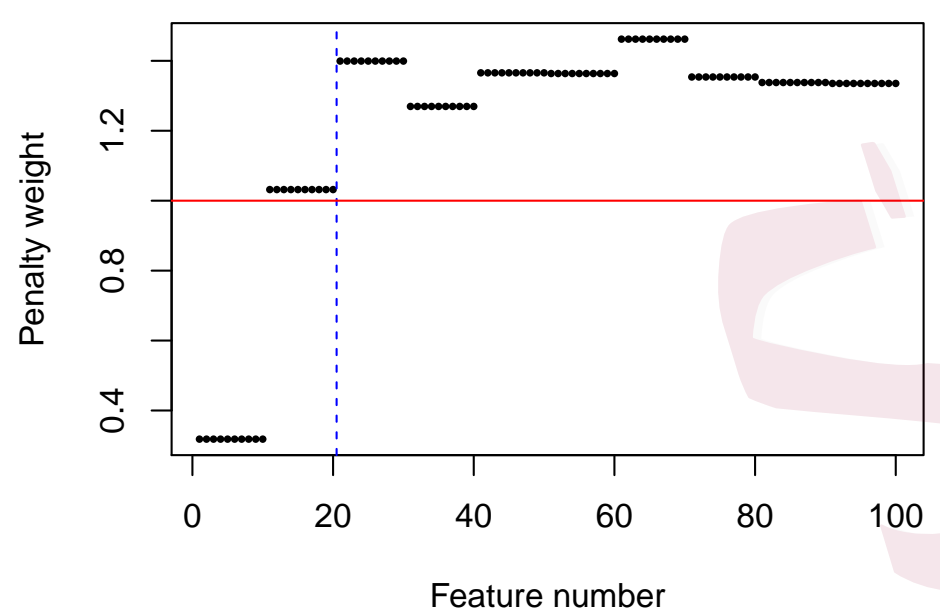

Figure 1: Penalty factors which fwelnet assigns to each feature. $n=200$, $p=100$ with features in groups of size 10. The response is a noisy linear combination of the first two groups, with signal in the first group being stronger than that in the second. As expected, fwelnet's penalty weights for the true features (left of blue dotted line) are lower than that for null features. The elastic net would assign all features a penalty factor of 1 (horizontal red line).

mization problem:

$$
\begin{aligned}
\underset{\beta\left(\lambda_{i}\right), \theta\left(\lambda_{i}\right)}{\operatorname{minimize}} \quad \frac{1}{m} \sum_{i=1}^{m} & {\left[\frac{1}{2}\left\|\mathbf{y}-\mathbf{X} \beta\left(\lambda_{i}\right)\right\|_{2}^{2}\right.} \\
& \left.+\lambda_{i} \sum_{j=1}^{p} w_{j}\left(\theta\left(\lambda_{i}\right)\right)\left(\alpha\left|\beta_{j}\left(\lambda_{i}\right)\right|+\frac{1-\alpha}{2} \beta_{j}\left(\lambda_{i}\right)^{2}\right)\right]
\end{aligned}
$$

subject to $\quad \theta\left(\lambda_{1}\right)=\cdots=\theta\left(\lambda_{m}\right)$, 
3.1 Computing the fwelnet solution11

where $\lambda_{1}>\lambda_{2}>\cdots>\lambda_{m}$ is a path of $\lambda$ hyperparameter values. Here, we think of $\theta$ as an argument of the objective function $J$, and we view minimizing $J$ as a joint function of $\beta$ and $\theta$ as a heuristic to obtain a good value of $\theta$. However, to maintain the interpretation of $\theta$ as a hyperparameter, we force $\hat{\theta}$ to be the same across all $\lambda$ values. We propose an alternating minimization (Algorithm 1) to solve this minimization problem. Step 3(c) finds the optimum solution for $\beta\left(\lambda_{1}\right), \ldots, \beta\left(\lambda_{m}\right)$ for given values of $\theta\left(\lambda_{1}\right), \ldots, \theta\left(\lambda_{m}\right)$, while Steps 3(a) and 3(b) does gradient descent for $\theta\left(\lambda_{1}\right), \ldots, \theta\left(\lambda_{m}\right)$ projected to the constraint set.

Because of the backtracking line search in Step 3(b) and the fact that Step 3(c) solves a convex problem, Algorithm 1 is guaranteed to converge, albeit to a stationary point. However, because Step 2 initializes $\hat{\beta}\left(\lambda_{i}\right)$ at the elastic net coefficients, we usually end up with a good solution. In our simulations, convergence was almost always reached within 20 iterations, and often one to three passes gave a sufficiently good solution.

Remark: We also considered an approach where $\theta$ was not constrained to be the same across $\lambda$ values. While conceptually straightforward, the algorithm was computationally slow and did not perform as well as Algorithm 1 in prediction. A sketch of this approach is in Appendix S1.

We have developed an $\mathrm{R}$ package, fwelnet, which implements Algo- 


\section{Algorithm 1 Fwelnet algorithm}

1. Select a value of $\alpha \in[0,1]$ and a sequence of $\lambda$ values $\lambda_{1}>\ldots>\lambda_{m}$.

2. For $i=1, \ldots, m$, initialize $\beta^{(0)}\left(\lambda_{i}\right)$ at the elastic net solution for the corresponding $\lambda_{i}$. Initialize $\theta^{(0)}=\mathbf{0}$.

3. For $k=0,1, \ldots$ until convergence:

(a) Set $\Delta \theta$ to be the component-wise mean of $\left.\frac{\partial J_{\lambda_{i}, \alpha}}{\partial \theta}\right|_{\beta=\beta^{(k)}, \theta=\theta^{(k)}}$ over $i=1, \ldots, m$.

(b) Set $\theta^{(k+1)}=\theta^{(k)}-\eta \Delta \theta$, where $\eta$ is the step size computed via backtracking line search to ensure that the mean of $J_{\lambda_{i}, \alpha}\left(\beta^{(k)}, \theta^{(k+1)}\right)$ over $i=1, \ldots, m$ is less than that for $J_{\lambda_{i}, \alpha}\left(\beta^{(k)}, \theta^{(k)}\right)$.

(c) For $i=1, \ldots, m$, set $\beta^{(k+1)}\left(\lambda_{i}\right)=$ elastic net solution for $\lambda_{i}$ where the penalty factor for feature $j$ is $w_{j}\left(\theta^{(k+1)}\right)$. 
3.2 Extending fwelnet to generalized linear models (GLMs)13

rithm 1. Step 3(c) of Algorithm 1 can be done easily by using the glmnet function in the glmnet $\mathrm{R}$ package and specifying the penalty.factor option. In practice, we use the sequence $\lambda_{1}>\cdots>\lambda_{m}$ provided by glmnet's implementation of the elastic net as this range of $\lambda$ values covers a sufficiently wide range of models. (In our package, we allow the user to replace the component-wise mean with the component-wise median in Step 3(a), and to replace the mean with the median in Step 3(b). We find these options do not change performance much when the default sequence is used, so we recommend that users stick with the defaults.)

\subsection{Extending fwelnet to generalized linear models (GLMs)}

It is easy to extend the elastic net to generalized linear models (GLMs) by replacing the RSS term with the negative log-likelihood of the data:

$$
\left(\hat{\beta}_{0}, \hat{\beta}\right)=\underset{\beta_{0}, \beta}{\operatorname{argmin}} \sum_{i=1}^{n} \ell\left(y_{i}, \beta_{0}+\sum_{j=1}^{p} x_{i j} \beta_{j}\right)+\lambda \sum_{j=1}^{p}\left[\alpha\left|\beta_{j}\right|+\frac{1-\alpha}{2} \beta_{j}^{2}\right],
$$

where $\ell\left(y_{i}, \beta_{0}+\sum_{j} x_{i j} \beta_{j}\right)$ is the negative log-likelihood contribution of observation $i$. Fwelnet can be extended to GLMs in a similar fashion:

$$
\left(\hat{\beta}_{0}, \hat{\beta}, \hat{\theta}\right)=\underset{\beta_{0}, \beta, \theta}{\operatorname{argmin}} \sum_{i=1}^{n} \ell\left(y_{i}, \beta_{0}+\sum_{j=1}^{p} x_{i j} \beta_{j}\right)+\lambda \sum_{j=1}^{p} w_{j}(\theta)\left[\alpha\left|\beta_{j}\right|+\frac{1-\alpha}{2} \beta_{j}^{2}\right],
$$

with $w_{j}(\theta)$ as defined in (3.1). Algorithm 1 can be used as-is to solve (3.4). 
Because $\theta$ only appears in the penalty term, this extension can be implemented easily. We can rely on glmnet for Steps 2 and 3(c), Step 3(a) is the same as before, and Step 3(b) simply requires a function that allows us to compute $\ell$.

\section{Theoretical connections}

\subsection{Connection to the group lasso}

One common setting where "features of features" arise naturally is when the features come in non-overlapping groups. Assume that the features in $\mathbf{X}$ come in $K$ non-overlapping groups. Let $p_{k}$ denote the number of features in group $k$, and let $\beta^{(k)}$ denote the subvector of $\beta$ which belongs to group $k$. Assume also that $\mathbf{y}$ and the columns of $\mathbf{X}$ are centered so that $\hat{\beta}_{0}=0$. In this setting, Yuan and Lin (2006) introduced the group lasso estimate as the solution to the optimization problem

$$
\underset{\beta}{\operatorname{minimize}} \frac{1}{2}\|\mathbf{y}-\mathbf{X} \beta\|_{2}^{2}+\lambda \sum_{k=1}^{K}\left\|\beta^{(k)}\right\|_{2} .
$$

The $\ell_{2}$ penalty on features at the group level ensures that features belonging to the same group are either all included in the model or all excluded from it. Often, the penalty given to group $k$ is modified by a 
factor of $\sqrt{p_{k}}$ to take into account varying group sizes:

$$
\hat{\beta}_{g l, 2}(\lambda)=\underset{\beta}{\operatorname{argmin}} \frac{1}{2}\|\mathbf{y}-\mathbf{X} \beta\|_{2}^{2}+\lambda \sum_{k=1}^{K} \sqrt{p_{k}}\left\|\beta^{(k)}\right\|_{2} .
$$

Theorem 1 below establishes a connection between fwelnet and the group lasso.

Theorem 1. If the "features of features" matrix $\mathbf{Z} \in \mathbb{R}^{p \times K}$ is given by $z_{j k}=1\{$ feature $j \in$ group $k\}$, then minimizing the fwelnet objective function (3.2) jointly over $\beta_{0}, \beta$ and $\theta$ reduces to

$$
\begin{aligned}
& \underset{\beta}{\operatorname{argmin}} \frac{1}{2}\|\mathbf{y}-\mathbf{X} \beta\|_{2}^{2}+\lambda^{\prime} \sum_{k=1}^{K} \sqrt{p_{k}\left[\alpha\left\|\beta^{(k)}\right\|_{1}+\frac{1-\alpha}{2}\left\|\beta^{(k)}\right\|_{2}^{2}\right]} \\
& =\left\{\begin{array}{lll}
\underset{\beta}{\operatorname{argmin}} & \frac{1}{2}\|\mathbf{y}-\mathbf{X} \beta\|_{2}^{2}+\lambda^{\prime} \sum_{k=1}^{K} \sqrt{p_{k}}\left\|\beta^{(k)}\right\|_{2} & \text { if } \alpha=0, \\
\underset{\beta}{\operatorname{argmin}} & \frac{1}{2}\|\mathbf{y}-\mathbf{X} \beta\|_{2}^{2}+\lambda^{\prime}\left(\sum_{k=1}^{K} \sqrt{p_{k}\left\|\beta^{(k)}\right\|_{1}}\right)^{2} & \text { if } \alpha=1,
\end{array}\right.
\end{aligned}
$$

for some $\lambda^{\prime} \geq 0$.

We recognize the $\alpha=0$ case as minimizing the RSS and the group lasso penalty, while the $\alpha=1$ case is minimizing the RSS and the $\ell_{1}$ version of the group lasso penalty. The proof of Theorem 1 can be found in Appendix S2

\subsection{Connection to Bayesian estimation}

Regularized estimators can often be thought of as the Bayes posterior mode for a given prior distribution. For example, it is well-known that if the prior 
and likelihood are given by

$$
\beta \stackrel{i . i . d .}{\sim} \mathcal{N}\left(0, \tau^{2} \mathbf{I}\right), \quad \mathbf{y} \mid \mathbf{X}, \beta \sim \mathcal{N}\left(\mathbf{X} \beta, \sigma^{2} \mathbf{I}\right)
$$

for some $\tau^{2}, \sigma^{2}>0$, then the posterior distribution for $\beta$ is minimized at the ridge regression solution for $\lambda=\sigma^{2} /\left(2 \tau^{2}\right)$. If feature information is available, a better prior might be one where the $\beta_{j}$ are exchangeable conditional on the $\mathbf{z}_{j}$ 's, i.e. $\beta_{j} \stackrel{i . i . d .}{\sim} G\left(\cdot \mid \mathbf{z}_{j}\right)$ for some prior distribution $G$. One possible choice is

$$
\beta_{j} \stackrel{i n d}{\sim} \mathcal{N}\left(0, v_{j}^{2} \tau^{2}\right), \quad v_{j}^{2}=\frac{p \exp \left(\mathbf{z}_{j}^{T} \theta\right)}{\sum_{\ell=1}^{p} \exp \left(\mathbf{z}_{\ell}^{T} \theta\right)}
$$

for some fixed $\theta$. With this prior, $\tau^{2}$ is the average prior variance for the $\beta_{j}$ 's, while the $v_{j}^{2}$ 's modulate the prior variance for each coefficient based on its feature information. The expression for the $v_{j}^{2}$ 's is simply softmax applied to the $\mathbf{z}_{j}^{T} \theta$ 's (scaled by $p$ ), a commonly used function to convert a vector of real values to a probability vector. Features with larger scores $\mathbf{z}_{j}^{T} \theta$ have correspondingly larger $v_{j}^{2}$, meaning they are more likely to have larger coefficients in the model. Straightforward computation shows that the posterior mode for $\beta$ is the fwelnet solution $(3.2)$ with $\alpha=0$ and $\lambda=$ $\sigma^{2} /\left(2 \tau^{2}\right)$. Algorithm 1 can be viewed as an empirical Bayes approximation to estimate $\theta$. For other values of $\alpha$, the fwelnet solution corresponds to 
the posterior mode for the following prior on $\beta$ :

$$
p\left(\beta_{j}\right) \propto \exp \left[-v_{j}^{2} \tau^{2}\left(\alpha\left|\beta_{j}\right|+\frac{1-\alpha}{2} \beta_{j}^{2}\right)\right], \quad v_{j}^{2}=\frac{p \exp \left(\mathbf{z}_{j}^{T} \theta\right)}{\sum_{\ell=1}^{p} \exp \left(\mathbf{z}_{\ell}^{T} \theta\right)} .
$$

This connection also presents a way to incorporate feature information in a fully Bayesian framework: instead of estimating $\theta$ from the data, we could impose a prior on it. This direction also gives us an explicit way to encode beliefs about the relative importance of the sources of side information for the predictive model.

\section{A simulation study}

We tested the performance of fwelnet against other methods in a simulation study. In the three settings studied, the true signal is a linear combination of the columns of $\mathbf{X}$, with the true coefficient vector $\beta$ being sparse. The response $\mathbf{y}$ is the signal corrupted by additive Gaussian noise. In each setting, we gave different types of feature information to fwelnet to determine the method's effectiveness.

For all methods, we used cross-validation (CV) to select the tuning parameter $\lambda$. Unless otherwise stated, the $\alpha$ hyperparameter was set to 1 (i.e. no $\ell_{2}$ squared penalty). To compare methods, we considered the mean squared error $(\mathrm{MSE}) M S E=\mathbb{E}\left[(\hat{y}-\mu)^{2}\right]$ achieved on 10,000 test points, as well as the true positive rate (TPR) and false positive rate (FPR) of the 
5.1 Setting 1: Noisy version of the true $|\beta| 18$

fitted models. ( $\hat{y}$ denotes the model's prediction while $\mu$ denotes the true underlying signal. The oracle model which knows the true coefficient vector $\beta$ can compute $\mu$ exactly and hence has a test MSE of 0 .) We ran each simulation 30 times to get estimates for these quantities. (See Appendix S3 for details of the simulations.)

\subsection{Setting 1: Noisy version of the true $|\beta|$}

In this setting, we have $n=100$ observations and $p=50$ features, with the true signal being a linear combination of just the first 10 features. The feature information matrix $\mathbf{Z}$ has a single column: a noisy version of $|\beta|$.

We compared fwelnet against the lasso (using the glmnet package) and the adaptive lasso (using the OLS solution as the pilot estimator) across a range of signal-to-noise ratios (SNR) in both the response $\mathbf{y}$ and the feature information matrix $\mathbf{Z}$ (see details in Appendix S3.1). The results are shown in Figure 2. As we would expect, the test MSE figures for the methods decreased as the SNR in the response increased. Fwelnet performed the best, with improvement over the other methods increasing as the SNR in $\mathbf{Z}$ increased, up to a point. In terms of feature selection, fwelnet appeared to have similar TPR but smaller FPR. 
5.1 Setting 1: Noisy version of the true $|\beta| 19$

\section{Boxplot of Test MSE}
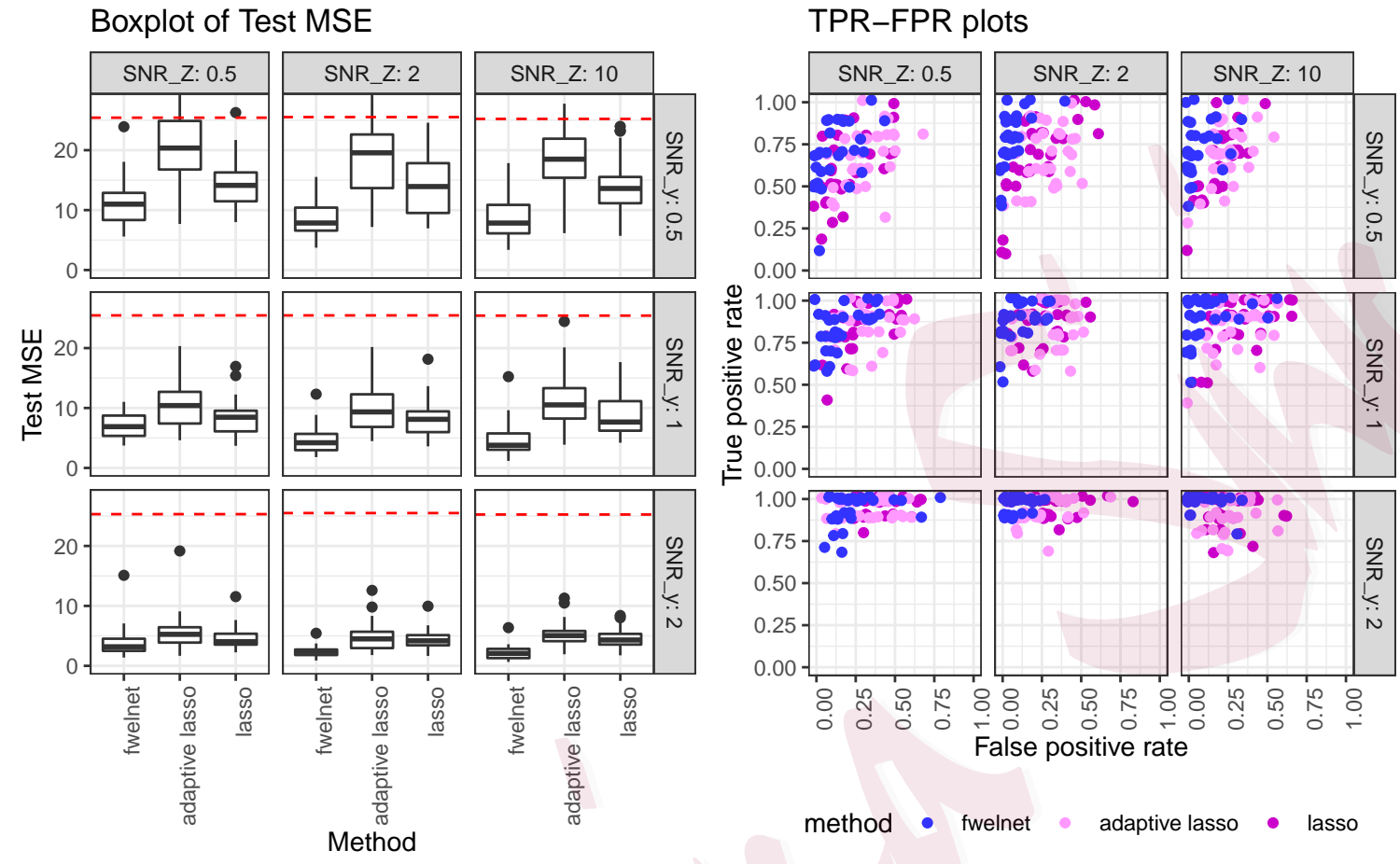

Figure 2: "Feature of features": noisy version of the true $|\beta| . \quad n=100$, $p=50$. The response is a linear combination of the first 10 features. As we go from left to right, the signal-to-noise ratio (SNR) for $\mathbf{y}$ increases; as we go from top to bottom, the SNR in $\mathbf{Z}$ increases. The left panel shows the test mean squared error (MSE) figures with the red dotted line indicating the median null test MSE. In the figure on the right, each point depicts the true positive rate (TPR) and false positive rate (FPR) of the fitted model for one of 30 simulation runs. Fwelnet performs the best in test MSE, with the improvement getting larger as the SNR in $\mathbf{Z}$ increases, up to a point. Fwelnet appears to have similar TPR but significantly smaller FPR. 


\subsection{Setting 2: Grouped data setting}

In this setting, we have $n=100$ observations and $p=150$ features, with the features coming in 15 groups of size 10. The feature information matrix $\mathbf{Z} \in \mathbb{R}^{150 \times 15}$ contains group membership information for the features: $z_{j k}=1\{$ feature $j \in$ group $k\}$. We compared fwelnet against the lasso, the adaptive lasso, and the group lasso (using the grpreg package) across a range of signal-to-noise ratios (SNR) in the response $\mathbf{y}$. (For the adaptive lasso, we used the lasso solution with $\lambda$ chosen by $\mathrm{CV}$ as the pilot estimator, since the OLS solution is unidentified in this setting.)

We considered two different responses in this setting. The first response was a linear combination of the features in the first group only, with additive Gaussian noise. The results are depicted in Figure 3. In terms of test MSE, fwelnet was competitive with the group lasso in the low SNR scenario and edged out the group lasso slightly for the highest SNR setting. In terms of feature selection, fwelnet had comparable TPR as the group lasso (except in the lowest SNR setting) but drastically smaller FPR. Fwelnet had better TPR and FPR than the lasso in this case.

The second response was not as sparse in the features: the true signal was a linear combination of the first 4 feature groups. The results are shown in Figure 4. In this case, fwelnet with $\alpha$ fixed at 1 lags the group lasso 
Boxplot of Test MSE

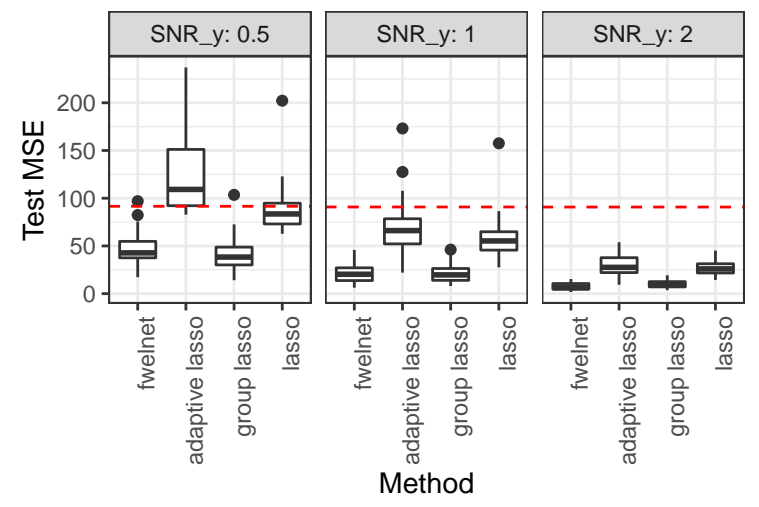

TPR-FPR plots

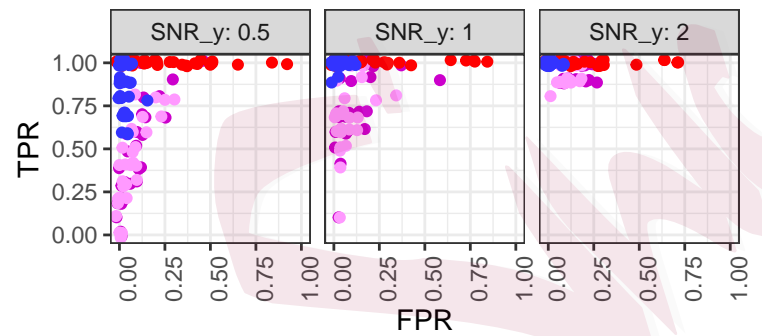

- fwelnet adaptive lasso

- group lasso - lasso

Figure 3: "Feature of features": grouping data. $n=100, p=150$. The features come in groups of 10, with the response being a linear combination of the features in the first group. As we go from left to right, the signalto-noise ratio (SNR) for $\mathbf{y}$ increases. The figure on the left shows the test mean squared error (MSE) results with the red dotted line indicating the median null test MSE. In the figure on the right, each point depicts the true positive rate (TPR) and false positive rate (FPR) of the fitted model for one of 30 simulation runs. Fwelnet performs comparably to the group lasso in terms of test MSE. Fwelnet has higher TPR than the lasso, and lower FPR than the group lasso. 
5.2 Setting 2: Grouped data setting22

slightly in test MSE. It is worth noting that fwelnet with $\alpha=1$ performs appreciably better than the lasso when the SNR is higher. Selecting $\alpha$ via cross-validation improved the test MSE performance of fwelnet slightly but not enough to outperform the group lasso; it also came at the cost of very high FPR.

\section{Boxplot of Test MSE}

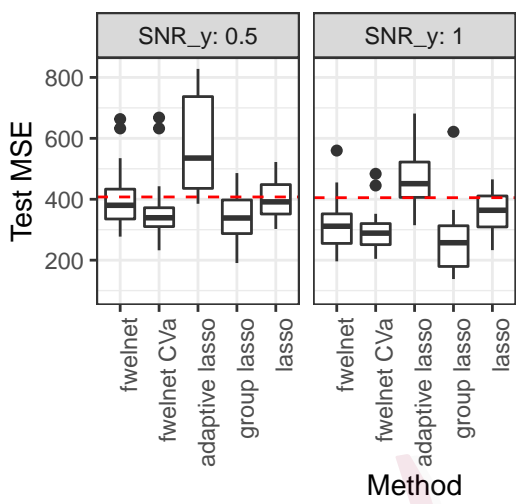

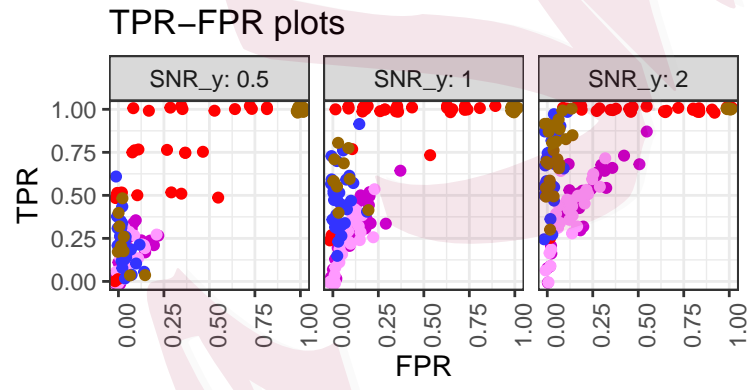

- fwelnet fwelnet CVa - adaptive lasso

- group lasso - lasso

Figure 4: "Feature of features": grouping data. $n=100, p=150$. The features come in groups of 10, with the response being a linear combination of the first 4 groups. As we go from left to right, the SNR for $\mathbf{y}$ increases. The left figure shows the test MSE results with the red dotted line indicating the median null test MSE. Fwelnet sets $\alpha=1$ while fwelnet $C V a$ selects $\alpha$ via cross-validation. In the figure on the right, each point depicts the TPR and FPR of the fitted model for one of 30 simulation runs. Group lasso performs best here. $C V$ for $\alpha$ improves test MSE performance slightly but at the expense of having very high FPR. 


\subsection{Setting 3: Noise variables}

In this setting, we have $n=100$ observations and $p=80$ features, with the true signal being a linear combination of just the first 10 features. The feature information matrix $\mathbf{Z}$ consists of 10 noise variables that have nothing to do with the response. Since fwelnet is adapting to these features, we expect it to perform worse than comparable methods.

We compare fwelnet against the lasso and the adaptive lasso (using the OLS solution as the pilot estimator): the results are depicted in Figure 5. As expected, fwelnet has higher test MSE than the lasso, but the decrease in performance is not drastic. The adaptive lasso performs much more poorly than the other methods. This is likely due to unstable least squares estimates for the weights due to $p$ being close to $n$. Fwelnet attained similar FPR and TPR to the lasso.

\section{Application: Early prediction of preeclampsia}

Preeclampsia is a leading cause of maternal and neonatal morbidity and mortality, affecting 5 to 10 percent of all pregnancies. The biological and phenotypical signals associated with late-onset preeclampsia strengthen during the course of pregnancy, often resulting in a clinical diagnosis after 20 weeks of gestation (Zeisler et al., 2016). An earlier test for late-onset 


\section{Boxplot of Test MSE}

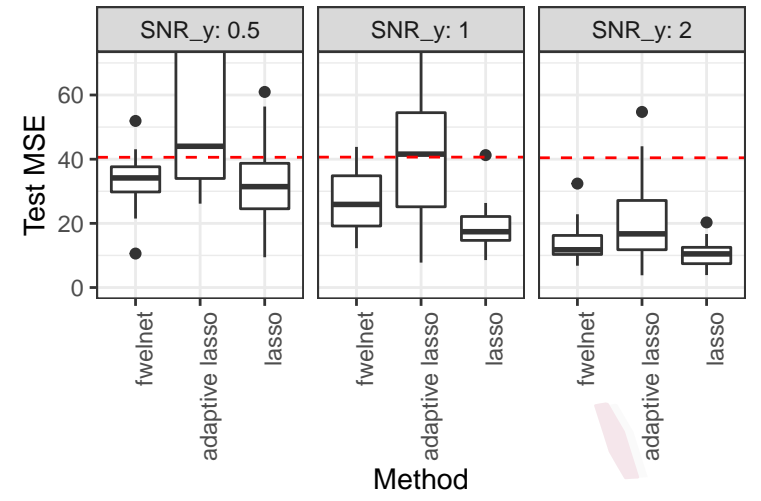

\section{TPR-FPR plots}

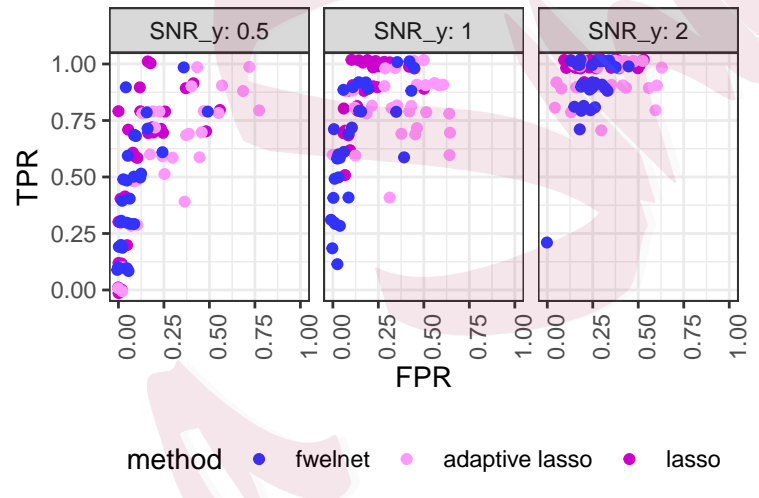

Figure 5: "Feature of features": 10 noise variables. $n=100, p=80$. The response is a linear combination of the first 10 features. Going from left to right, the SNR for $\mathbf{y}$ increases. The left figure shows the test MSE results, with the red dotted line indicating the median null test MSE. In the right figure, each point depicts the TPR and FPR of the fitted model for one of 30 simulation runs. Fwelnet only performs slightly worse than the lasso in test MSE, and has similar TPR and FPR as the lasso. 
preeclampsia has substantially higher clinical value as it enables interventions for improvement of maternal and neonatal outcomes Jabeen et al. 2011). In this example, we leverage protein data collected in late pregnancy, which is closer to the onset of preeclampsia but of lower clinical utility, to learn about the proteins most helpful for this prediction task, then use this information to build a model using protein measurements from early in the pregnancy. It is important to note that data from late pregnancy is only used to train the model: for prediction on new patients, only the samples collected during early pregnancy will be needed.

We used a dataset of 1,125 plasma proteins measured during various gestational ages of pregnancy (Erez et al. 2017). The SOMAScan platform used in this dataset produces targeted measurements of a broad range of proteins that are broadly related to various aspects of human biology. To maintain the exploratory nature of the study, we did not select specific proteins that are expected to be related to preeclampsia based on prior studies. We considered time points $\leq 20$ weeks "early" and time points $>20$ weeks as "late". The dataset consisted of 166 patients each having 2 to 6 time points, for a total of 666 time point observations. Protein measurements were log-transformed to reduce skewness. We used the following procedure to build a predictive model based on early time point data only: 
1. Patients were split randomly into two equal-sized buckets. For patients in the first bucket, we only used their late time points (83 patients with 219 time points) while for patients in the second bucket, we only used their early time points (83 patients with 116 time points).

2. We trained an elastic net logistic regression model on the late time points for patients in the first bucket to predict whether the patient would have preeclampsia (using the log-transformed protein measurements for predictors). $\alpha$ was set to 0.5 and $\lambda$ was selected by crossvalidation $(\mathrm{CV})$. We extracted model coefficients at the $\lambda$ value which gave the highest $\mathrm{CV}$ area under the curve (AUC).

3. We trained a fwelnet logistic regression model on the early time points for patients in the second bucket, using the absolute values of the late time point model coefficients as feature information. $\alpha$ was set to 1 and we computed the 10-fold CV AUC for the entire path of $\lambda$ values.

When performing CV in Steps 2 and 3, we made sure that observations from one patient all belonged to the same CV fold to avoid "contamination" of the held-out fold. One can also run the fwelnet model with additional sources of feature information for each of the proteins.

Figure 6 shows a plot of 10-fold CV AUC for the fwelnet model in Step 
3 and the baseline lasso model against the number of features in the model. The lasso obtains a maximum CV AUC of 0.80 , while fwelnet obtains the largest CV AUC of 0.84 .

In running the workflow several times, we noted that the results were somewhat dependent on (i) how the patients were split into the two buckets in Step 1, and (ii) how patients were split into CV folds when training the models in Steps 2 and 3. We found that if the late time point model had few non-zero coefficients, then the fwelnet model for early time point data was very similar to that for the lasso. This matches our intuition: few nonzero coefficients means injecting very little additional information through fwelnet's relative penalty factors. Nevertheless, we did not encounter cases where running fwelnet gave worse CV AUC than the lasso.

\section{Using fwelnet for multi-task learning}

We turn now to an application of fwelnet to multi-task learning. Here, we have a single model matrix $\mathbf{X}$ but are interested in multiple responses $\mathbf{y}_{1}, \ldots \mathbf{y}_{B}$. If there is some common structure between the signals in the responses, it can be advantageous to fit models for them simultaneously. This is especially useful if the responses have low SNR.

We demonstrate how fwelnet can be used to learn better models in 


\section{0-fold CV AUC vs. model size}

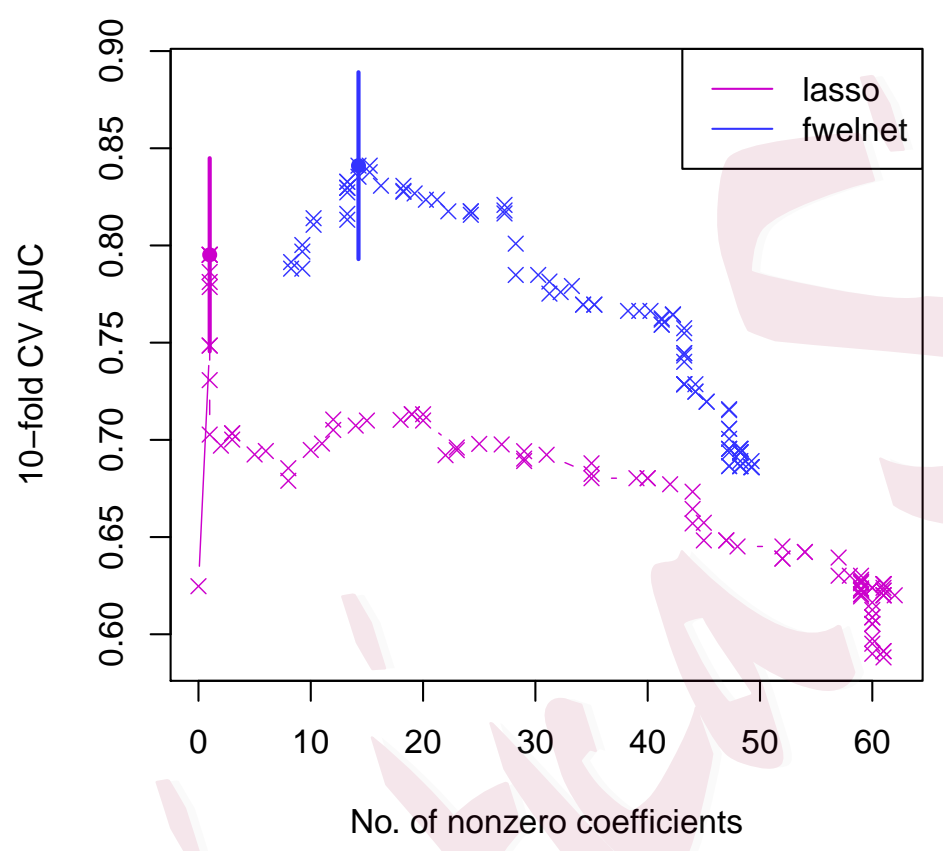

Figure 6: Early prediction of preeclampsia: Plot of 10-fold cross-validated $(C V)$ area under the curve (AUC). 10-fold CV AUC is plotted against the number of non-zero coefficients for each model trained on just early time point data. For each method, the model with highest $C V A U C$ is marked by a dot. To reduce clutter in the figure, the \pm 1 standard error bars are drawn for just these models. Fwelnet achieved higher CV AUC for the same model size, i.e. number of features with non-zero coefficients. 
the setting with two responses, $\mathbf{y}_{1}$ and $\mathbf{y}_{2}$. The idea is to use the absolute value of coefficients of one response as the external information for the other response. That way, a feature which has larger influence on one response is likely to be given a correspondingly lower penalty weight when fitting the other response. Algorithm 2 presents one possible way of doing so.

\section{Algorithm 2 Using fwelnet for multi-task learning}

1. Initialize $\beta_{1}^{(0)}$ and $\beta_{2}^{(0)}$ at the lambda.min elastic net solutions for $\left(\mathbf{X}, \mathbf{y}_{1}\right)$ and $\left(\mathbf{X}, \mathbf{y}_{2}\right)$ respectively, that is, the value of the hyperparameter $\lambda$ which minimizes cross-validated error.

2. For $k=0,1, \ldots$ until convergence:

(a) Set $\mathbf{Z}_{2}=\left|\beta_{1}^{(k)}\right|$. Run fwelnet with $\left(\mathbf{X}, \mathbf{y}_{2}, \mathbf{Z}_{2}\right)$ and set $\beta_{2}^{(k+1)}$ to be the lambda.min solution.

(b) Set $\mathbf{Z}_{1}=\left|\beta_{2}^{(k+1)}\right|$. Run fwelnet with $\left(\mathbf{X}, \mathbf{y}_{1}, \mathbf{Z}_{1}\right)$ and set $\beta_{1}^{(k+1)}$ to be the lambda.min solution.

We tested the effectiveness of Algorithm 2 (with step 2 run for 3 iterations) on simulated data. We generate 150 observations with 50 independent features. The signal in response 1 is a linear combination of features 1 to 10 , while the signal in response 2 is a linear combination of features 1 
to 5 and 11 to 15 . The coefficients are set such that those for the common features (i.e. features 1 to 5) have larger absolute value than those for the features specific to one response. The signal-to-noise ratios (SNRs) in response 1 and response 2 are 0.5 and 1.5 respectively. (See Appendix S4 for more details of the simulation.)

We compared Algorithm 2 against: (i) the individual lasso (ind_lasso), where the lasso is run separately for $\mathbf{y}_{1}$ and $\mathbf{y}_{2}$; and (ii) the multi-response lasso (mt_lasso) (Obozinski et al., 2010), where coefficients belonging to the same feature across the responses are given a joint $\ell_{2}$ penalty. Because of the $\ell_{2}$ penalty, a feature is either included or excluded in the model for all the responses at the same time.

Figure 7 shows the results for 50 simulation runs. Fwelnet outpeforms the other two methods in test MSE as evaluated on 10,000 test points. The individual lasso performs well for the higher SNR response but poorly for the lower SNR response. The multi-response lasso is able to borrow strength from the higher SNR response to obtain good performance on the lower SNR response. However, because the models for both responses are forced to have the same set of features, performance suffers on the higher SNR response. Fwelnet has the ability to borrow strength across responses without being hampered by this restriction. 

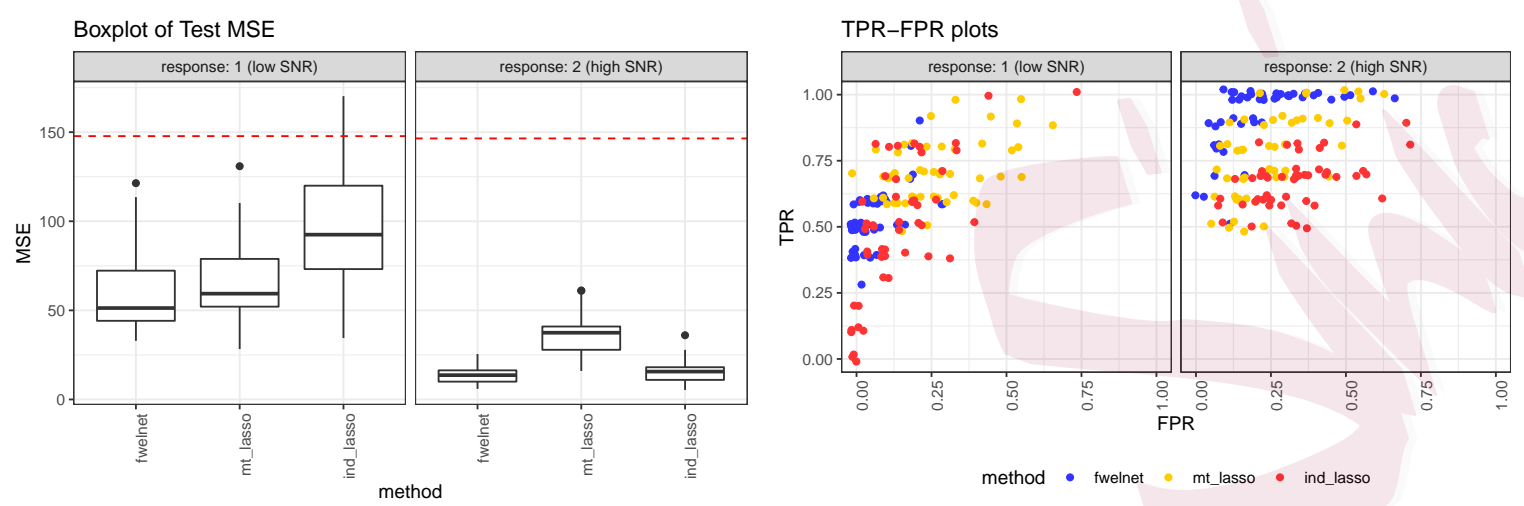

Figure 7: Application of fwelnet to multi-task learning. $n=150, p=50$.

Response 1 is a linear combination of features 1 to 10, while response 2 is a linear combination of features 1 to 5 and 11 to 15 . The SNR for the responses are 0.5 and 1.5 respectively. The left figure shows the test MSE figures with the red dotted line indicating the median null test MSE. The right figure shows the TPR and FPR of the fitted model (each point being one of 50 simulation runs). Fwelnet outperforms the individual lasso and the multi-response lasso in test MSE for both responses. Fwelnet also appears to have better FPR than the other methods and better TPR than the individual lasso. 


\section{Discussion}

In this paper, we have proposed a method for exploiting external information about predictor variables. We do this by organizing these "features of features" as a matrix $\mathbf{Z} \in \mathbb{R}^{p \times K}$, and modifying model-fitting algorithms by assigning each feature a score, $\mathbf{z}_{j}^{T} \theta$, based on this auxiliary information. We have proposed one such method, the feature-weighted elastic net ( fwelnet"), which imposes a penalty modification factor $w_{j}(\theta)=\frac{\sum_{\ell=1}^{p} \exp \left(\mathbf{z}_{\ell}^{T} \theta\right)}{p \exp \left(\mathbf{z}_{j}^{T} \theta\right)}$ for the elastic net algorithm.

This method is widely applicable in that there are no restrictions on the type of feature information that can be incorporated into $\mathbf{Z}$ as long as it is real-valued. As such, we recommend using fwelnet whenever feature information is available (e.g. grouping information, prior guesses on feature importance). When the feature information is relevant to the prediction problem, in that it has some signal on how important a feature is to predicting the response, we expect fwelnet to outperform competitor methods. At the same time, simulation setting 3 (Section 5.3) shows that using irrelevant feature information can be detrimental to the fit. In practice we recommend using domain knowledge to guide selection of side information for the model. We also recommend fitting the vanilla elastic net and comparing the CV error of the two methods: this comparison will show if the 
feature information was relevant to the prediction problem or not.

There is much scope for future work:

- Interpretation of $\mathbf{z}_{j}^{T} \theta$ and $\theta$. As noted in the Introduction, $\mathbf{z}_{j}^{T} \theta$ can be thought of as an indication of how influential feature $j$ is on the response, since a larger $\mathbf{z}_{j}^{T} \theta$ corresponds to a smaller penalty weight $w_{j}(\theta)$ (see Equations (3.1) and (3.2)).

The interpretation for $\theta$ is not as straightforward. When $\mathbf{Z} \in \mathbb{R}^{p \times K}$ is orthonormal, we can interpret $\theta_{k}$ as the relative importance of the $k$ th source of feature information for identifying important features for the prediction problem. However, this interpretation becomes less clear when there are correlations between the columns of $\mathbf{Z}$. In the extreme case where there is multicollinearity in $\mathbf{Z}, \theta$ is not identified even though $\mathbf{z}_{j}^{T} \theta$ is unique. These are the same issues one faces when interpreting OLS coefficients in the presence of feature correlations.

- Different choices of side information $\mathbf{Z}$. We have explored a few different choices of side information, including prior coefficient estimates and group membership. It would be interesting to evaluate fwelnet's effectiveness when using other types of side information. One natural extension of group membership is probabilistic group membership, 
where each feature is assigned a probability distribution across the $K$ groups. Another extension is overlapping groups, where each row of $\mathbf{Z}$ need not sum to 1 . $\mathbf{Z}$ as a $p \times p$ similarity matrix is another option which can be thought of as a combination of the two extensions above, with group $j$ being associated with feature $j$, and the degree of group membership measured by how similar each feature is to feature $j$.

- Whether $\theta$ should be treated as a parameter or a hyperparameter, and how to determine its value. We introduced $\theta$ as a hyperparameter for (3.2). This gives us a clear interpretation for $\theta$ described above. However, grid search computation to find its optimal value grows exponentially with the number of sources of feature information. To avoid this growth, we suggested a descent algorithm for $\theta$ based on its gradient with respect to the fwelnet objective function. There are other methods for hyperparameter optimization such as random search (e.g. Bergstra and Bengio (2012)) or Bayesian optimization (e.g. Snoek et al. (2012)) that could be applied.

One could consider $\theta$ as an argument of the fwelnet objective function to be minimized over jointly with $\beta$. This approach gives us a theoretical connection to the group lasso (Section 4.1). However, we will obtain different estimates of $\theta$ for each value of the hyperparameter 
$\lambda$, which may be undesirable for interpretation. The objective function is also not jointly convex in $\theta$ and $\beta$, so different minimization algorithms could end up at different local minima. Our attempts to make this approach work (see Appendix S1) did not fare as well in prediction performance and was computationally expensive.

- Choice of penalty modification factor. While the penalty modification factor $w_{j}(\theta)$ we have proposed works well in practice and has several desirable properties, we make no claim about its optimality.

- Extending the use of scores beyond the elastic net. The use of feature scores $\mathbf{z}_{j}^{T} \theta$ in modifying feature weights is a general idea that could apply to any supervised learning algorithm. More work needs to be done on how such scores can be incorporated, with particular focus on how $\theta$ can be learned through the algorithm.

An $R$ language package $f$ welnet which implements our method is available at https://www.github.com/kjytay/fwelnet.

\section{Supplementary Materials}

The online supplementary materials provide (i) details on an alternative algorithm with $\theta$ as a parameter, (ii) details on the simulation study in Section 5, (iii) the proof for Theorem 1, and (iv) details on the simulation 
study in Section 7 .

\section{Acknowledgements}

We would like to thank Nikolaos Ignatiadis, the associate editor and reviewers for helpful discussions and comments which improved the quality of our paper. Nima Aghaeepour was supported by the Bill \& Melinda Gates Foundation (OPP1189911, OPP1113682), the National Institutes of Health (R01AG058417, R01HL13984401, R61NS114926, R35GM138353-01), the Burroughs Wellcome Fund and the March of Dimes Prematurity Research Center at Stanford University. Trevor Hastie was partially supported by the National Science Foundation (DMS-1407548 and IIS1837931) and the National Institutes of Health (5R01 EB001988-21). Robert Tibshirani was supported by the National Institutes of Health (5R01 EB001988-16) and the National Science Foundation (19 DMS1208164).

\section{References}

Bergersen, L. C., I. K. Glad, and H. Lyng (2011). Weighted lasso with data integration. Statistical Applications in Genetics and Molecular Biology 10(1).

Bergstra, J. and Y. Bengio (2012). Random search for hyper-parameter optimization. Journal of Machine Learning Research 13, 281-305.

Boulesteix, A.-L., R. De Bin, X. Jiang, and M. Fuchs (2017). IPF-LASSO: Integrative L1- 


\section{REFERENCES37}

Penalized Regression with Penalty Factors for Prediction Based on Multi-Omics Data. Computational and Mathematical Methods in Medicine 2017, 1-14.

Erez, O., R. Romero, E. Maymon, P. Chaemsaithong, B. Done, P. Pacora, B. Panaitescu, T. Chaiworapongsa, S. S. Hassan, and A. L. Tarca (2017). The prediction of late-onset preeclampsia: Results from a longitudinal proteomics study. PLoS ONE 12(7), e0181468.

Friedman, J., T. Hastie, and R. Tibshirani (2010). Regularization Paths for Generalized Linear Models via Coordinate Descent. Journal of Statistical Software 33(1), 1-24.

Hoerl, A. E. and R. W. Kennard (1970). Ridge regression: Biased estimation for nonorthogonal problems. Technometrics 12(1), 55-67.

Jabeen, M., M. Y. Yakoob, A. Imdad, and Z. A. Bhutta (2011). Impact of interventions to prevent and manage preeclampsia and eclampsia on stillbirths. BMC Public Health 11 (S3), S6.

Jacob, L., G. Obozinski, and J.-P. Vert (2009). Group Lasso with overlap and graph Lasso. In Proceedings of the 26th annual international conference on machine learning, pp. 433-440.

Li, C. and H. Li (2008). Network-constrained regularization and variable selection for analysis of genomic data. Bioinformatics 24(9), 1175-1182.

Mollaysa, A., P. Strasser, and A. Kalousis (2017). Regularising non-linear models using feature side-information. Proceedings of the 34th International Conference on Machine Learning, $2508-2517$. 


\section{REFERENCES38}

Obozinski, G., B. Taskar, and M. I. Jordan (2010). Joint covariate selection and joint subspace selection for multiple classification problems. Statistics and Computing 20(2), 231-252.

Slawski, M., W. zu Castell, and G. Tutz (2010). Feature selection guided by structural information. Annals of Applied Statistics 4(2), 1056-1080.

Snoek, J., H. Larochelle, and R. P. Adams (2012). Practical Bayesian optimization of machine learning algorithms. In Advances in neural information processing systems, pp. 2951-2959.

Tai, F. and W. Pan (2007). Incorporating prior knowledge of predictors into penalized classifiers with multiple penalty terms. Bioinformatics 23(14), 1775-1782.

Tibshirani, R. (1996). Regression Shrinkage and Selection via the Lasso. Journal of the Royal Statistical Society. Series B (Methodological) 58(1), 267-288.

Tibshirani, R., M. Saunders, S. Rosset, J. Zhu, and K. Knight (2005). Sparsity and smoothness via the fused lasso. Journal of the Royal Statistical Society: Series B (Statistical Methodology) 67(1), 91-108.

van de Wiel, M. A., T. G. Lien, W. Verlaat, W. N. van Wieringen, and S. M. Wilting (2016). Better prediction by use of co-data: adaptive group-regularized ridge regression. Statistics in Medicine 35(3), 368-381.

Velten, B. and W. Huber (2018). Adaptive penalization in high-dimensional regression and classification with external covariates using variational Bayes. arXiv preprint arXiv:1811.02962. 


\section{REFERENCES39}

Yuan, M. and Y. Lin (2006). Model Selection and Estimation in Regression with Grouped Variables. Journal of the Royal Statistical Society. Series B (Statistical Methodology) 68(1), $49-67$.

Zeisler, H., E. Llurba, F. Chantraine, M. Vatish, A. C. Staff, M. Sennström, M. Olovsson, S. P. Brennecke, H. Stepan, D. Allegranza, P. Dilba, M. Schoedl, M. Hund, and S. Verlohren (2016). Predictive value of the sFlt-1:PlGF ratio in women with suspected preeclampsia. New England Journal of Medicine 374(1), 13-22.

Zou, H. (2006). The adaptive lasso and its oracle properties. Journal of the American Statistical Association 101 (476), 1418-1429.

Zou, H. and T. Hastie (2005). Regularization and variable selection via the elastic net. Journal of the Royal Statistical Society: Series B (Statistical Methodology) 67(2), 301-320.

\section{J. Kenneth Tay: Department of Statistics, Stanford University}

E-mail: kjytay@stanford.edu

Nima Aghaeepour: Department of Anesthesiology, Pain, and Perioperative Medicine, Stanford University, Department of Pediatrics, Stanford University, and Department of Biomedical Data Sciences, Stanford University

E-mail: naghaeep@stanford.edu

Trevor Hastie: Department of Statistics, Stanford University, and Department of Biomedical

Data Sciences, Stanford University 
E-mail: hastie@stanford.edu

Robert Tisbshirani: Department of Statistics, Stanford University, and Department of Biomedical Data Sciences, Stanford University

E-mail: tibs@stanford.edu 